\title{
Astronomia no Infravermelho de Estrelas Variáveis
}

\author{
Infrared Astronomy of Variable Stars \\ C.E.F. Lopes* e M.G. Pereira ${ }^{\dagger}$ \\ Departamento de Física - UEFS \\ Campus Universitário, Km 03, BR 116 \\ Feira de Santana - BA - 44031-4660
}

\begin{abstract}
Este trabalho tem por objetivo fazer um estudo fotométrico de estrelas variáveis utilizando dados fotométricos do projeto 2 Micron All Sky Survey. Foram analisados dados de fotometria infravermelho nas bandas JHKs de cinco tipos de estrelas variáveis: Wolf Rayet, RS Canum Venaticorum, Sistemas Binários de Raios-X, Fontes Super Soft e Estrelas Simbióticas. As materializações de diagramas CorCor permitiram um estudo das propriedades fotométricas infravermelho intrínsecas destas fontes, e assim obteve-se uma ferramenta para identificação de alvos para estudos mais detalhados a serem realizados posteriormente com telescópios de maior porte.
\end{abstract}

Palavras-chaves: Astronomia, Fotometria, Estrelas Variáveis, Diagrama Cor-Cor, Infravermelho.

\begin{abstract}
This work has the objective to do a study about photometry of variable stars by using photometric data from project 2 Micro All Sky Survey. We make an analysis of data in the infrared photometry to JHKs gaps of five variable stars types: Wolf Rayet, RS Canum Venaticorum, X-Rays Binary Systems, Super Soft Sources and Symbiotic Stars. The Color-Color diagram representations allow to study of intrinsic infrared photometric proprieties of these sources, and so we get a tool for the target identification to more detailed studies to realize in future time through the use of more powerful telescopes.
\end{abstract}

Key-words: Astronomy, Photometry, Variable Stars, Color-Color Diagram, Infrared.

\section{INTRODUÇÃO}

O infravermelho é uma região do espectro eletromagnético que nos últimos tempos tem apresentado grande desenvolvimento na astronomia, com o advento das matrizes de detectores que vem possibilitando o imageamento de grandes regiões do céu.

Nesta região do espectro, processos associados com a emissão térmica dos objetos estelares têm sido pouco estudados devido às baixas sensibilidades dos detectores, devido à forte contaminação do ruído térmico, e baixas resoluções espectrais, usualmente prejudicadas pelas dificuldades de se construírem unidades de matrizes de detector com dimensões $1 \mu \mathrm{m}$.

Com o advento dos grandes telescópios optimizados para o infravermelho (Gemini e SOAR, por exemplo), tem-se a necessidade de se estabelecer metodologias para a determinação de alvos a serem observados por estes telescópios.

Projetos de varreduras do céu nesta região do espectro tem sido realizados, como o DENIS e 2MASS (2 Micron All Sky Survey).

Este artigo é parte de um projeto desenvolvido para estudar as propriedades fotométricas intrínsecas das estrelas variáveis na região espectral do infravermelho próximo $(0,78$ até $3 \mu m)$ utilizando os dados públicos do projeto 2MASS. Consiste na obtenção de magnitudes nas bandas fotométricas do infravermelho $J H K s$, e a partir destas medidas obter os índices de cor $J-H$ e $H-K s$, para a construção dos diagramas Cor-Cor. Com estes diagramas esperamos obter uma forma fácil e direta de identificarmos objetos peculiares, assim como efetuarmos classificação de classes que apresentam "locus" comum dentro dos diagramas Cor-Cor.

\section{METODOLOGIA DE AQUISIÇÃO}

O trabalho, objetivando observar estrelas variáveis no infravermelho, passa por uma fase de escolha dos alvos a partir dos quais serão feitas as obtenções de imagens para identificação, e a posterior realização da fotometria para obtenção das magnitudes JHKs. Após a realização da fotometria, são calculados os índices de cor $J-H$ e $H-K$. Entretanto, essas cores não representam as cores intrínsecas de cada fonte, tendo em vista que estas magnitudes estão afetadas pelo avermelhamento, sendo necessário então, fazer um levantamento de como varia esta extinção com a distância. Também são usadas estrelas padrões fotométricas no infravermelho [1], para comparação entre as magnitudes de outros observatórios e as magnitudes do $2 M A S S$. Para efeitos de comparação das magnitudes 2MASS e estrelas desavermelhadas, foi feito um levantamento de estrelas anãs e gigantes desavermelhadas [2], as quais são apresentadas como referência em todos os diagramas cor-cor, de modo a se obter uma ferramenta de observação direta do quanto algum objeto se desvia da seqüência principal.

\footnotetext{
* Electronic address: carlos.eduardo@uefs.br

$\dagger$ Electronic address: marildo1@uefs.br
}

\section{TELESCÓPIO E CÂMERAS}


O $2 M A S S$ é um projeto de mapeamento de todo céu em três faixas do infravermelho próximo, composto por dois telescópios refletores idênticos em sua construção. Cada um dos telescópios do $2 M A S S$ é composto foco Cassegrain, montagem equatorial, espelho primário de 1,3 metros de diâmetro, banda passante $\mathrm{J}$ 1,11 até 1,36 $\mu \mathrm{m}-\mathrm{H}$ 1,50 até $1,80 \mu m$ - K 2,00 até $2,32 \mu m$ e por três detectores NICMOS3 $256 \times 256$, os quais podem obter imagens simultâneas nas bandas infravermelho centradas em $1.25 m(J), 1.65 m(H)$, e $2.17 m(K s)$. Um dos telescópios fica situado no Mt. Hopkins no Arizona, e o outro em Cerro Tolo no Chile. Os dados do projeto $2 M A S S$ estão disponíveis para a comunidade astronômica, podendo ser obtidos no endereço na web [14].

\section{OBTENDO OS DADOS E IMAGENS FOTOMÉTRICAS}

O Instituto de Tecnologia da Califórnia (CalTech), com apoio da Agencia Espacial Americana $(N A S A)$, criou o Centro de Processamento e Análise no Infravermelho do CalTech (Infrared Processing and Analysis Center at CalTech - IPAC), que tem como nodo principal o Arquivo de Ciência no Infravermelho (Infrared Science Archive IRSA), o qual é totalmente voltado para missões e projetos no infravermelho. Este projeto pode ser acessado no endereço eletrônico http://irsa.ipac.caltech.edu/index.html, e a partir desta interface obter as informações e dados das várias missões astronômicas dirigidas no infravermelho.

Os dados de imagens e fotometria no infravermelho obtidos para estudo neste trabalho, são oriundos do projeto 2MASS, e foram obtidos a partir de duas ferramentas:

a) Interactive $2 M A S S$ All-Sky Quicklook Image Service - Serviço voltado para o fornecimento de imagens nas bandas JHKs com uma interface simples, na qual os dados de entrada são as coordenadas da posição do objeto e a dimensão da imagem. O endereço de acesso desta interface é http://irsa.ipac.caltech.edu/applications2MASS/QL/ interactive.html.

b) GATOR INTERFACE - Esta interface permite que o usuário faça pesquisas em vários dos catálogos contidos no banco de dados NASA/IPAC. A interface permite em sua primeira pagina selecionar o catálogo desejado, no caso deste trabalho às fontes estavam ligadas ao 2MASS All-Sky Point Source Catalog (PSC) (Catálogo 2MASS de fontes pontuais). Selecionado o catálogo, segue-se para a segunda pagina, que é o local onde se seleciona a posição da fonte, o raio de busca da fotometria, magnitudes nas bandas $J H K s$, entre outras informações, disponibilizados na web no endereço http://irsa.ipac.caltech.edu/applications/Gator/.

\section{FOTOMETRIA INFRAVERMELHA}

A fotometria é uma técnica que permite medir em um detector, o número de fótons provenientes de uma certa fonte astrofísica, de modo a ser obtida a magnitude de uma fonte. As bandas espectrais usadas no infravermelho são construídas de modo a usar as janelas de transmissão atmosférica, tendo em vista que grande parte da radiação nesta região do espectro sofre poucos efeitos de absorção.

As medidas fotométricas podem ser realizadas a partir de imagens obtidas com detectores infravermelhos. Uma vez obtidas estas imagens, devem ser descontados os efeitos de ruído térmico, não linearidade e contaminação do fundo de céu e radiação térmica do ambiente. Uma vez realizadas estas rotinas preliminares, são definidas aberturas circulares em torno dos alvos, de modo a medirmos a contribuição das fontes e do céu vizinho a estas. Uma vez subtraída esta contribuição de céu, é obtido o valor das contagens no detector, os quais são usados para a obtenção da magnitude da fonte observada. Para efeitos de calibração, são observadas também estrelas padrões, de forma a permitir uma determinação absoluta dos valores das magnitudes medidas.

\section{FONTES ESTUDADAS}

São denominadas estrelas variáveis aquelas estrelas em que a variação não representa apenas as flutuações normais de grandes conjuntos de partículas em movimentos turbulentos, mas apresentam amplitudes mensuráveis com certo grau de regularidade. A variação de brilho pode ocorrer devido a fatores intrínsecos da própria estrutura física das estrelas, a estas chamamos de variáveis intrínsecas, como também em decorrência de fatores externos, eclipses parciais ou totais devido ao fato da estrela pertencer a um sistema estelar binário ou múltiplo, estas são denominadas variáveis geométricas ou eclipsantes.

As classes de estrelas variáveis analisadas neste trabalho compreendem variáveis do tipo; RS Canum Venaticorum $(R S C V n)$, Wolf Rayet $(W R), \mathrm{X}$-Ray Binary $(S B R X), \mathrm{Su}-$ per Soft Source $(S S S)$ e Symbiotic Star, perfazendo um total de 751 estrelas.

\section{A. Estrelas Padrões}

Em física existe uma constante necessidade de estabelecermos quantidades que possam ser usadas como padrões de medida, em astronomia não é diferente, e para isto, usamos as chamadas estrelas padrões. Estas estrelas padrões devem apresentar brilho estável em longas escalas de tempo, devendo ter também propriedades fotométricas e espectroscópicas bem definidas, sendo assim, usadas para fins de assegurar a estabilidade de medidas fotométricas e a uniformidade dos níveis de calibração instrumental dos diferentes detectores usados nos observatórios. As estrelas padrões usadas neste trabalho compreendem estrelas com magnitudes absolutas intrínsecas bem definidas, como as gigantes e anãs desavermelhadas de Bessell e Brett, as quais são usadas para definir o local da seqüência principal desavermelhada nos diagramas Cor-Cor. Também são usadas as padrões fotométricas do UKIRT, Hubble e NOAO. Deste modo, em todos os diagramas Cor-Cor, são apresentadas as estrelas padrões e seus respectivos tipos espectrais. 


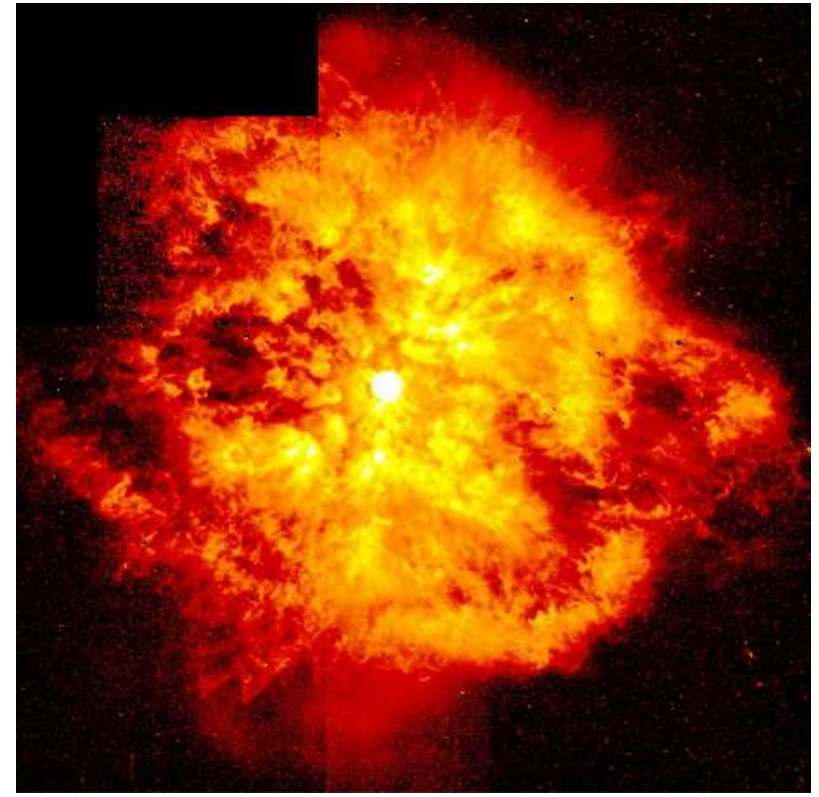

Fig. 1 - Estrela $W R(W R 124)$ da constelação da Águia, situada a uma distância de 15000 anos-luz do Sol. (Crédito: Hubble Space Telescope.)

\section{B. Estrelas do tipo Wolf-Rayet}

O Termo "Wolf-Rayet" (WR) refere-se a um tipo particular de espectro estelar que Wolf e Rayet (1867) descobriram enquanto observavam estrelas da constelação do Cisne. Estes espectros mostravam algumas linhas de emissão relativamente largas sobrepostas num espectro contínuo fraco. As estrelas do tipo $W R$ são objetos ricos em $H e$, sendo formadas por estrelas com massas superiores a 20 Massas Solares que perderam enormes quantidades de matéria por emissão de um vento estelar intenso, o que deixa suas regiões centrais praticamente "nuas". Esta não é, entretanto, a única explicação possível para a existência das estrelas $W R$, uma outra possibilidade é um mecanismo de troca de matéria em um sistema binário.

\section{Estrelas Simbióticas}

As estrelas Simbióticas são geralmente aceitas como sendo sistemas binários de longo período orbital $(\mathrm{P} \sim 200$ dias a 10 anos). Elas compreendem uma pequena classe de variáveis eruptivas que mostram um espectro simultâneo de estrela fria (forte contínuo vermelho, banda de absorção TIO e VO, linhas em absorção de metais ionizados simples neutros) e de uma componente quente (linhas em emissão da serie Balmer, HeI e [OIII] e linhas de emissão altamente ionizadas como HeII e [FeVII] e um fraco continuo azul.

O modelo binário consiste em uma gigante do tipo tardio $(G-M)$, ocasionalmente uma supergigante, transferindo material via vento estelar, ou por extravasamentos via preenchimento do Lóbulo de Roche pelo ponto interno de Lagrange, para um objeto compacto e quente. O perfil tipo tardio é associado com uma gigante vermelha e a estrela quente tem intensa radiação ultravioleta que ioniza uma parte da nebulosa a que envolve, dando assim origem a linhas de emissão.

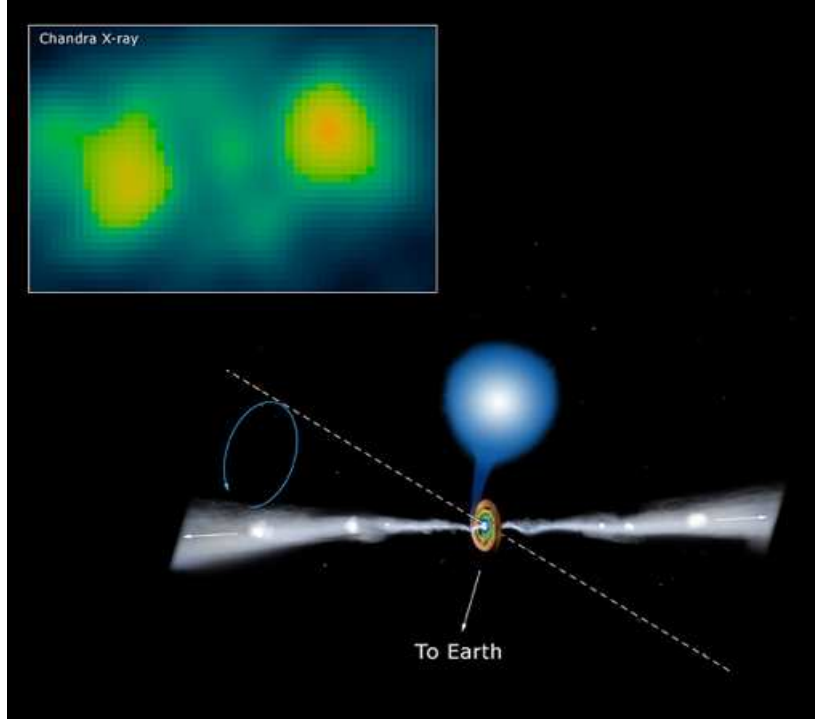

Fig. 2 - Imagem em raios-X e interpretação artística do Sistema Binário de Raios-X de alta massa SS433 (Crédito: X-ray: NASA/CXC/U.Amsterdam/S.Migliari et al., Illustration: CXC/M.Weiss)

\section{Sistemas Binários de Raios-X}

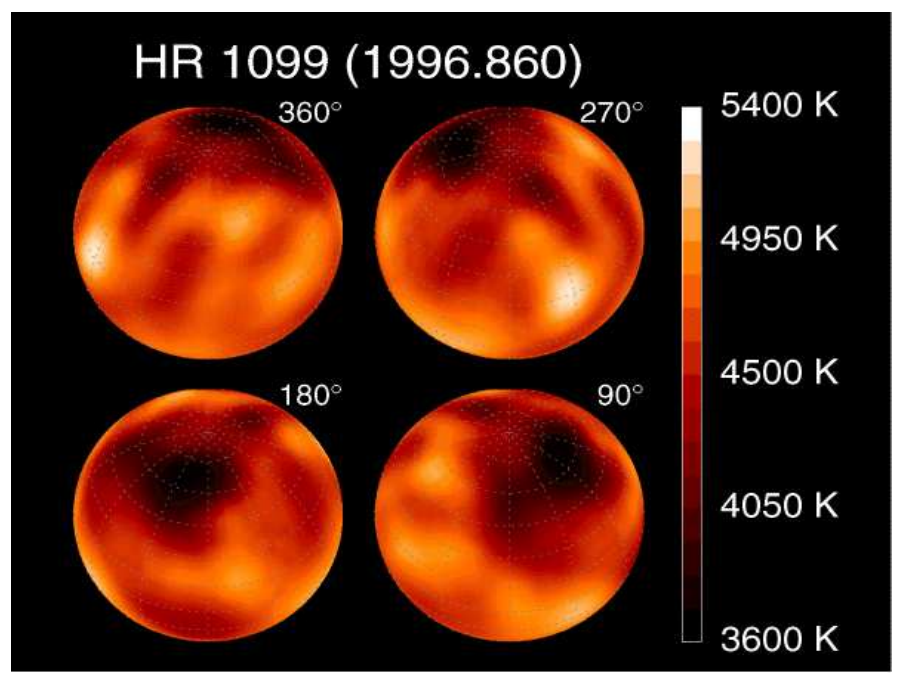

Fig. 3 - Modelo de uma estrela do tipo RS CVn (HR1099). A estrela apresenta manchas em sua atmosfera, as quais são responsáveis pela variação de sua curva de luz. (Crédito: http://astro.univie.ac.atjbartus[at]aip.deupdate: August 19, 2003)

Define-se um sistema binário de raios-X $(S B R X)$ como um sistema cerrado formado por uma estrela normal que transfere matéria para uma anã branca, estrela de nêutrons ou buraco negro. A transferência de matéria pode ocorrer na forma de vento estelar ou pelo transbordo do Lobo de Roche. A captura de matéria (usualmente chamada de disco de acréscimo) pelo objeto degenerado leva à produção de raios-X. Devido à força gravitacional do objeto compacto, a matéria da estrela companheira cai na sua direção, a fricção entre os diversos constituintes do gás é aquecida a temperaturas elevadas (100 milhões de graus ou mais) que este emite abundantemente nos comprimentos de onda 
mais energéticos, em particular nos raios-X com picos de energia entre $1-20 \mathrm{keV}$.

A variabilidade da radiação óptica e em raios-X pode ser classificada como regulares (pulsos e efeitos orbitais), semiregulares ("burst" e oscilações quase periódicas) e caótica (flares e flickering). Os $\sim 250 S B R X$ apresentam sua classificação baseada na massa da componente óptica de modo a serem agrupados em $S B R X$ de baixa massa $(B X B M)$ e de alta massa $(B X A M)$.

\section{Fontes Super Soft (SSS)}

Fontes $S S S$ são sistemas binários de raios-X, recentemente descobertos, que apresentam órbitas cerradas (com períodos $1 \mathrm{~d}$ ) em torno de uma estrela companheira com massa entre 1,2 - 2,5 massas solares. As $S S S$ diferem dos $S B R X$ por terem a sua fonte de raios-X com temperaturas $105 K$. O estudo do fluxo de radiação em raios-X emitida pelas SSS permitiu a determinação das dimensões da região emissora, chegando a um valor de aproximadamente $10000-20000 \mathrm{~km}$, ou seja, muito maior que as dimensões estimadas para uma estrela de nêutrons ou o "horizonte de eventos" de um buraco negro. Baseados nestas constatações e observações chegou-se à conclusão de que o objeto compacto responsável pela emissão da radiação só poderia ser uma anã branca, ou seja, uma estrela também degenerada, mas com densidades menores do que os buracos negros e estrelas de nêutrons.

\section{E. Fontes do tipo $R S 4$ Canum Venaticorum}

O sistema $R S C V n$ é tipicamente formado por uma estrela do tipo $F, G$ ou $K$, tendo como companheira uma estrela tardia que está entrando na seqüência principal ou uma sub-gigante.

As $R S C V n$ possuem períodos orbitais menores que 30 dias, apresentando usualmente sincronismo orbital e período rotacional, e $C A B S$ (Catalogue of Active Binary Star). Apresentam uma variação de luz devido as "manchas estelares", que podem ser análogos a classe de objetos do tipo BY Draconis (BY Dra), em função de manchas em sua superfície. Por outro lado, [3] sugere que os sistemas binários $R S C V n$ possam ser atualmente classificados como sistemas BY Dra.
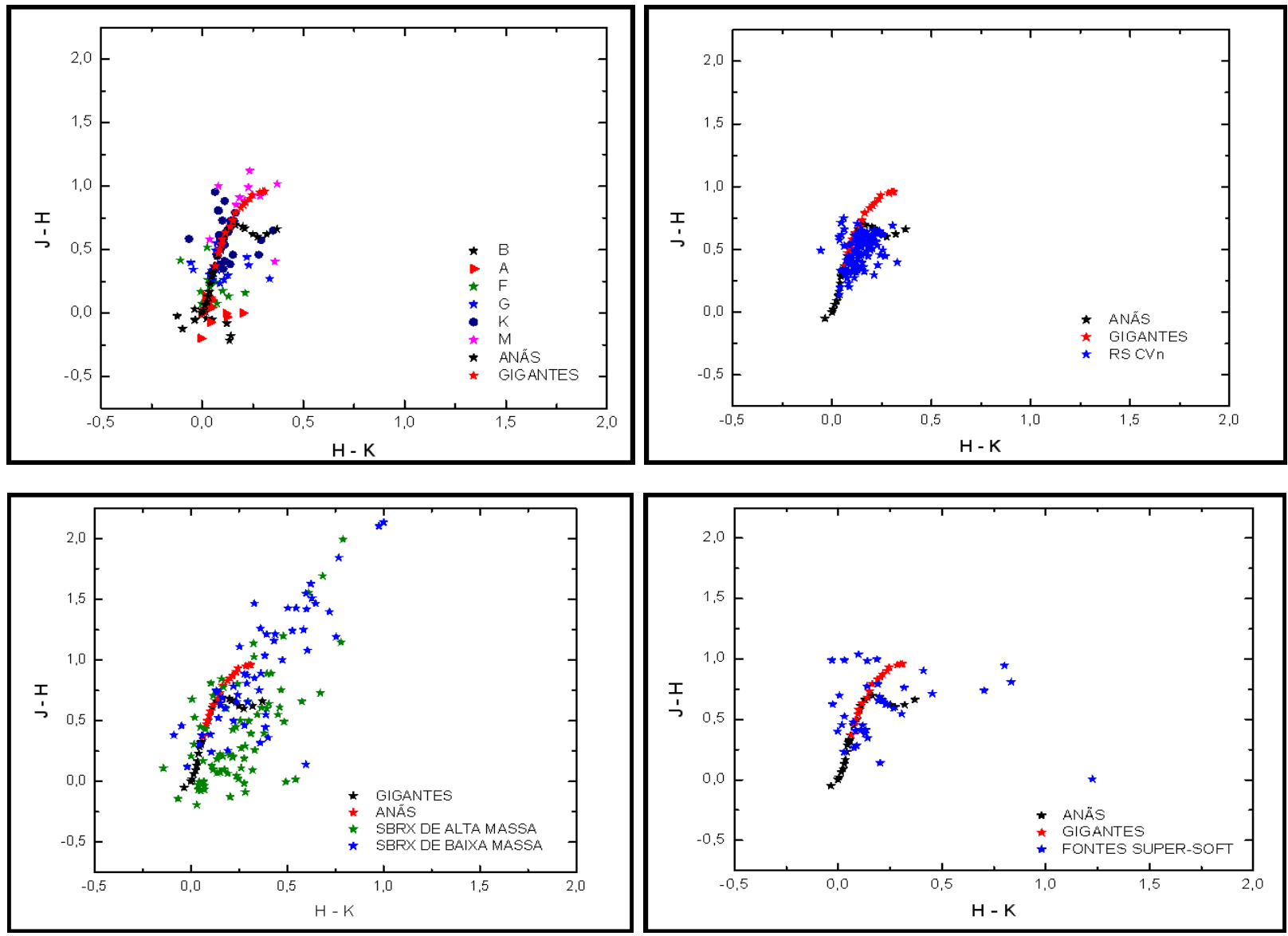

Fig. 4 - Diagramas Cor-Cor das estrelas das estrelas padrões, RS CVn, Sistemas Binários de Raios-X e Fontes Super Soft. Os pontos em preto e vermelho representam as estrelas gigantes e as anãs desavermelhadas de Bessell e Brett (1988), as quais são usadas para definir o local da seqüência principal nos diagramas Cor-Cor.

VII. DIAGRAMA COR-COR
Em um dado sistema de magnitudes, podem ser definidos os chamados índices de cor a partir de diferenças das magnitudes consideradas. Por exemplo, no sistema $U B V$ defi- 
nimos os índices $U-B$ e $B-V$. Tomando dois filtros quaisquer $A$ e $B$ pode ser definida a cor $A-B=m A-m B$. Estes índices são muito convenientes, variam de forma contínua e estão relacionados com as propriedades físicas intrínsecas das estrelas, em particular com sua temperatura, podendo ser computados usando uma aproximação de corpo negro. A validade desta aproximação pode ser aferida por um diagrama Cor-Cor envolvendo as cores $J-H$ e $H-K$, por exemplo. Este método permite um estudo das propriedades fotoelétricas intrínsecas das diferentes classes de variáveis, e assim obter uma ferramenta para identificação de alvos, os quais deverão ser objeto de estudo para determinação de suas origens. A seguir, são mostrados os diagramas CorCor, no caso deste trabalho $J-H \times H-K$, para 5 tipos de estrelas variáveis; $W R, R S C V n 4, S S S, X R B X$ e as estre- las Simbióticas. Em todos os diagramas são apresentadas, além das fontes alvos, as estrelas de comparação desavermelhadas anãs e gigantes [1].

Para efeito de não saturação dos diagramas Cor-Cor, apresentamos uma forma simples de visualizar os erros das magnitudes obtidas da fotometria $J H K s$ é através de um gráfico erro $\times$ magnitude. Este método, apos efetuarmos um ajuste de um polinômio, é aplicado para as três bandas fotométricas do infravermelho podendo ser observados na figura 6. Além do fato de que algumas classes de estrelas variáveis apresentam baixa amostragem, o uso deste ajuste permitiu a utilização de fontes nas quais o $2 M A S S$, devido a algum tipo de interferência, obteve a fotometria, porém não obteve o erro associado.
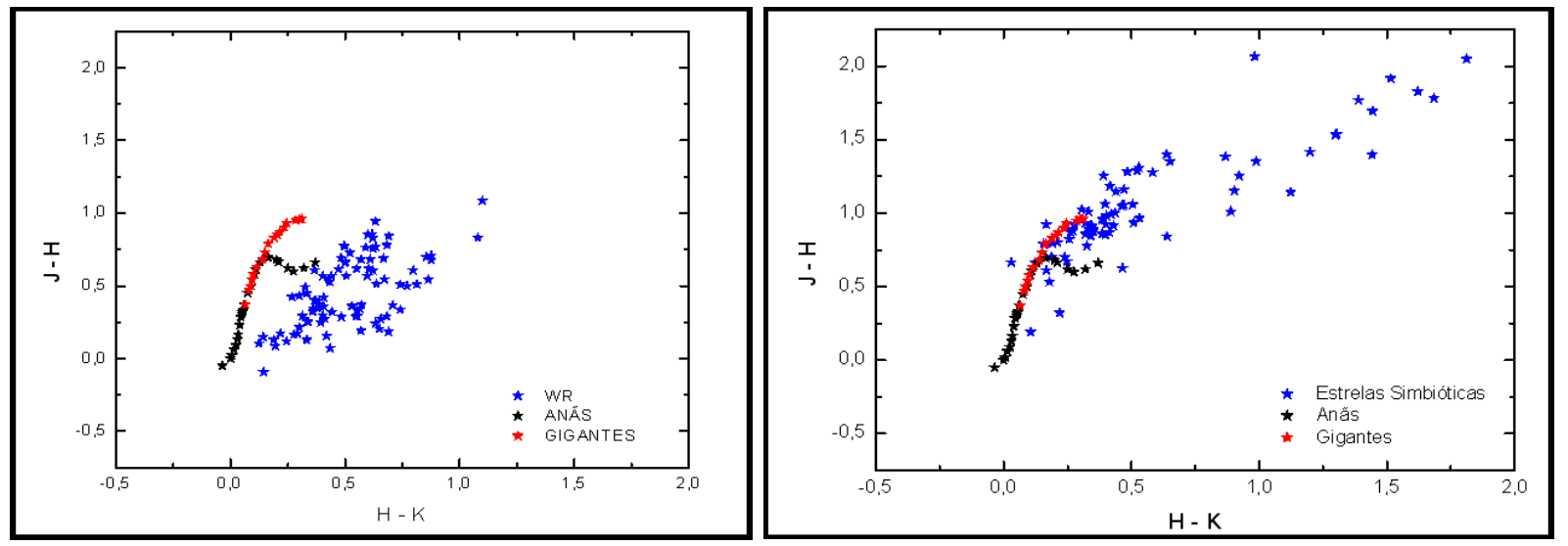

Fig. 5 - Diagramas Cor-Cor de cinco estrelas variáveis do tipo Wolf Rayet e Estrelas Simbióticas. Os pontos em preto e vermelho representam as estrelas gigantes e as anãs desavermelhadas de Bessell e Brett (1988), as quais são usadas para definir o local da seqüência principal nos diagramas Cor-Cor.

\section{CONCLUSÕES}

Este trabalho teve como objetivo o estudo de fontes astrofísicas associadas a estrelas variáveis, das quais foram obtidas fotometria no infravermelho nas bandas $J H K s$, provenientes do banco de dados do projeto $2 M A S S$. Foram analisadas 781 estrelas variáveis com magnitudes entre 4 e 16 nas bandas $J$ e $H 4$, e de 2 a 15 na banda $K s$, com incertezas máximas de $0,26-0,28 \mathrm{mag}$. O resumo das fontes analisadas é apresentado na Tabela 1.

No processo de obtenção dos diagramas de cores $J-H \times$ $H-K$ foram analisadas estrelas padrões que permitiram estabelecer a qualidade dos resultados a serem analisados. As estrelas padrões, as gigantes e as anãs desavermelhadas de Bessell \& Brett (1988), foram comparadas com estrelas padrões fotométricas, e ficaram em bom acordo com os valores médios dos vários tipos espectrais, indo em ordem decrescente de temperatura ( $\mathrm{O}$ a $\mathrm{M})$, com suas dispersões em torno da seqüência principal desavermelhada, mostrando o pequeno efeito de extinção no infravermelho, os quais são apresentados a seguir.

Em relação às estrelas variáveis, devem ser destacados os seguintes aspectos:

- Os Sistemas Binários de Raios-X apresentaram três regiões distintas: uma região inferior pertencente aos $S B R X$ de alta massa, associada a estrelas mais quentes; uma região superior povoada por SBRX de baixa massa com estrelas mais frias; e uma região intermediária entre os dois tipos de sistemas.

- As fontes $S S S$ mostram uma tendência de estarem próximas à seqüência principal, junto ao ramo das gigantes, em uma região similar às estrelas que apresentam propriedades intermediárias entre os $S B R X$ de alta massa e de baixa massa.

Podemos também inferir que existe uma subdivisão em classes das SSS, visto que, suas posições no diagrama de cores estão concentradas em três regiões aparentemente distintas.

De uma forma geral, o aspecto da distribuição das fontes $S S S$ é bastante semelhante ao observado no trabalho de Hoard, com as variáveis cataclísmicas.

- As estrelas Simbióticas ficaram deslocadas da seqüência principal na região do diagrama de estrelas mais frias, na parte superior do diagrama Cor-Cor. Objetos com avermelhamento intrínseco elevados ocupam as regiões mais elevadas do diagrama.

- As estrelas $R S C V n$ foram as que apresentaram menor dispersão, que é um indício de que estas fontes tenham propriedades muito semelhantes, se constituindo numa classe homogênea. As RSCVn localizaram-se na região de estrelas do tipo $F, G$ e 
$K$, indo bem de acordo com o modelo padrão para este tipo de fonte.

- As estrelas Wolf-Rayet sugerem a existência de duas classes: um ramo superior situado acima da bifurcação entre as anãs e gigantes desavermelhadas, com estrelas mais avermelhadas devido à poeira ao seu redor; e uma inferior, indicando temperaturas mais altas, baixo avermelhamento intrínseco, com núcleos mais expostos.
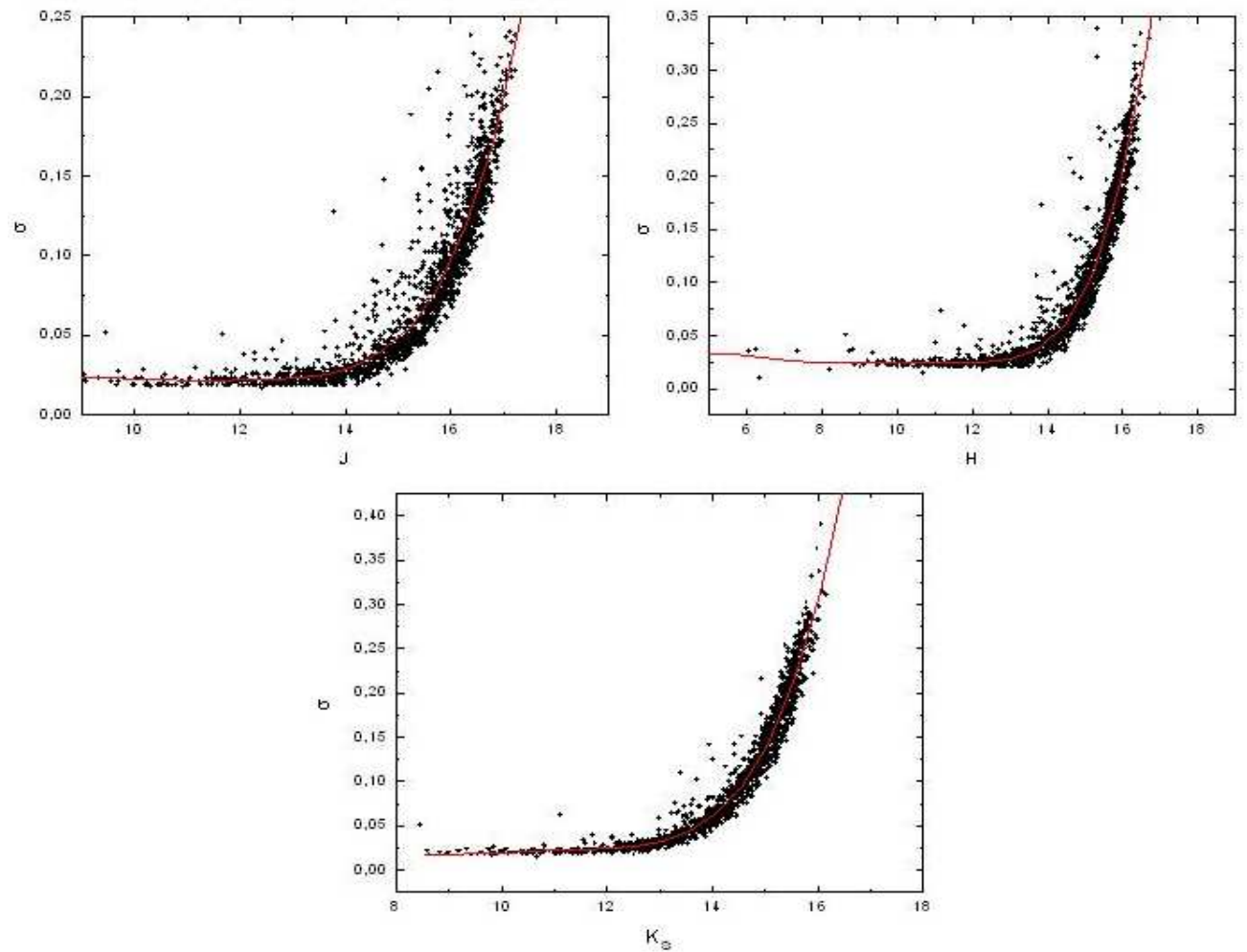

Fig. 6 - Os diagramas acima representam o comportamento do erro $(\sigma)$ nas bandas espectrais $J, H$ e $K s$ em função de suas respectivas bandas espectrais.

\begin{tabular}{|l|c|c|c|c|c|}
\hline Tipo & № & $\overline{J-H}$ & $\sigma_{J-H}$ & $\overline{H-K}$ & $\sigma_{H-K}$ \\
\hline WR & 226 & 0,512 & 0,197 & 0,459 & 0,238 \\
\hline SSS & 60 & 0,249 & 0,331 & 0,601 & 0,321 \\
\hline BXAM & 130 & 0,250 & 0,195 & 0,404 & 0,440 \\
\hline BXBM & 150 & 0,432 & 0,378 & 1,033 & 0,729 \\
\hline RS CVn & 80 & 0,137 & 0,062 & 0,480 & 0,127 \\
\hline Simbiótica & 135 & 0,563 & 0,425 & 1,060 & 0,367 \\
\hline
\end{tabular}

Tab. 1 - Expressa os valores médios de J-H e H-K, das estrelas variáveis estudadas, como as suas respectivas dispersões. Onde $\mathrm{N}^{\mathrm{o}}$ é o número de fontes estudadas.

De uma forma geral, a metodologia para a identificação e classificação de classes de estrelas variáveis se apre- senta muito promissora, em particular, os casos das estrelas $R S C V n$, Simbióticas e Wolf-Rayet. Os detalhes de diferenças intrínsecas dentro de classes que apresentam aparentes subdivisões (FontesSuperSoft e $S B R X$ ) trazem motivação para exames mais detalhados nas subclasses já existentes, além de indicar a necessidade de observações espectroscópicas para identificar a natureza intrínseca das fontes que contribuem para as cores observadas.

\section{AGRADECIMENTOS}

CEFL é suportado pela bolsa de iniciação científica PRO$\mathrm{BIC} / \mathrm{UEFS}$.
[1] J.E. Steiner e M.P. Diaz; PASP 110276 (1998).

[2] M.S. Bessell; et al., PASP 1001134 (1988).

[3] V.J. Maciel; Introdução à Estrutura e Evolução Estelar, Editora da Universidade de São Paulo, São Paulo (1999).

[4] J.H. Elias et al.; The Astronomy Journal 871029 (1982).

[5] J. Greiner et al.; New Astronomy 5 (3) 137 (2000).

[6] I.S. Glass; Handbook of Infrared Astronomy, Cambridge University Press, Cambridge (1999).
[7] C. Hoffmeister et al.; Variable Star, Springer-Verlag, Berlin (1984).

[8] D.W. Hoard, P. Szkody; Observations of Novalike Cataclysmic Variables with the Far Ultraviolet Spectroscopic Explorer, American Astronomical Society Meeting (2002).

[9] D.C. Murphy; The Astron. Journal 1162475 (1998).

[10] P. Kahabka et al.; Astronomy and Astrophysics 288538 (1994). 
[11] P. Kahabka et al.; Scientific American, 280 (2) (1999).

[12] M. Pereira et al.; Astronomia - Uma visão Geral do Universo, Editora da Universidade de São Paulo, São Paulo (2000).

[13] L. Wallace e K. Hinkle; Ap. 535325 (2000).
[14] http://irsa.ipac.caltech.edu

[15] http://chandra.harvard.edu/photo/2002/0214/0214 hand.html.

[16] http://adswww.harvard.edu/. 